\title{
Research Paper \\ Social Support, Responsibility, and Organizational Procrastination: A Mediator Role for Basic Psychological Needs Satisfaction
}

\author{
Parvaneh Amarloo', *Hossein Shareh ${ }^{2}$
}

1. MSc., Department of Psychology, Faculty of Humanities, Neyshabur Branch, Islamic Azad University, Neyshabur, Iran.

2. PhD, Associate Professor, Department of Educational Sciences, Faculty of Literature and Humanities, Hakim Sabzevari University, Sabzevar, Iran.

Received: 30 Jun 2017 Accepted: 12 Mar 2018

Key words: Need, Social support, Responsibility, Procrastination ceftat on: Amarloo P, Shareh H. [Social Support, Responsibility, and Organizational Procrastination: A Mediator Role for Basic Psychological Needs Satisfaction (Persian)]. Iranian Journal of Psychiatry and Clinical Psychology. 2018; 24(2):176-189. http://dx.doi.org/10.32598/ijpcp.24.2.176

http://dx.doi.org/10.32598/ijpcp.24.2.176

\section{ABSTRACT}

Objectives This study examined basic psychological needs satisfaction (i.e. the need for autonomy, competence and relatedness) as a mediator between social support, responsibility and organizational procrastination among school counselors.

Methods A descriptive correlational study design was used. Using the census method, all the school counselors of Sabzevar were selected. The subjects completed the Vaux Social Support Appraisals Scale (SS-A), Tuckman Procrastination Scale (TPS), responsibility subscale of the California Personality Inventory (CPI) and the Basic Needs Satisfaction in Relationships Scale.

Results There is a statistically significant negative relationship between components of social support and organizational procrastination $(P<0.05)$. Structural equation modeling analysis indicates that satisfaction of basic psychological needs mediates the relationship between social support and organizational procrastination $(\mathrm{P}<0.05)$. The mediator role of basic psychological needs satisfaction in the relationship between responsibility and organizational procrastination was not confirmed.

Conclusion Basic psychological needs satisfaction play a significant mediating role in the relationship between social support and organizational procrastination of the school counselors.

\section{Extended Abstract}

\section{Introduction}

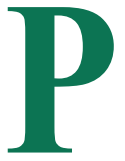

rocrastination is a gap between intention and behavior, characterized by doing less urgent tasks compared to more urgent ones, or preferring more pleasurable things over less pleasurable ones and thus postponing impending tasks for a later time. Procrastination in the long run affects a person's physical and mental health and is associated with many social and economic damages. Thus, it is important to understand what reduces procrastination and consequently decreases the psychological distress or sufferings of the individuals. Some researchers suggest that some factors such as irrational beliefs, self-esteem, self-prevention strategies, distress, anxiety and loneliness are predictor variables of procrastination. Multiple studies have considered social support as an important predictive factor for mental and physical health. Many investigations have demonstrated the association between procrastination and the component of social support. These studies have suggested social support as a factor that reduces job procrastination. In addition to the social support, responsible individuals are one of the factors reducing job procrastinations, since responsibility enables the individual to do more in minimum time by strengthening the attributes such as prudence, thoughtful actions, effective organization and

* Corresponding Author:

Hossein Shareh, PhD

Address: Department of Educational Sciences, Faculty of Literature and Humanities, Hakim Sabzevari University, Sabzevar, Iran.

Tel: +98 (51) 44410104

E-mail: hsharreh@yahoo.com.au 
optimal time management. Responsibility acts as a factor to satisfy the mutual needs of people in the society and promotes social solidarity. Basic psychological needs provide the necessary conditions for psychological growth, coherence and well-being of the people and explains the widespread underpinnings in the human behavior. It seems that basic psychological needs play an important role in job efficiency. This study aims to investigate the mediator role of basic psychological needs in the relationship between social support and responsibility with job procrastination among the school counselors.

\section{Method}

The present research is based on the structural equation model. All school counselors in Sabzevar were selected by the census method. It should be noted that the population size has been estimated to be 130 individuals according to the studies performed. Out of 130 counselors, 7 people refused to cooperate and the research sample was reduced to 123 subjects. The tools applied in this study included the Social Support Questionnaire (SSQ), Tuckman Procrastination Scale, Responsibility subscale of California Psychological Inventory and Basic Psychological Needs Satisfaction ScaleRelationship Domain. To analyze the data, structural equation analysis and path analysis methods were employed using the statistical software of SPSS-24 and AMOS-22.

\section{Results}

The results indicated that basic psychological needs play a significant mediating role in the relationship between social support and job procrastination $(\mathrm{P}<0.05)$. But basic psychological needs have no mediator role in the relationship between responsibility and job procrastination $(\mathrm{P}>0.05)$. Further, there was a significant relationship between social support and its components with job procrastination $(\mathrm{P}<0.05)$, but no significant relationship was found between responsibility and job procrastination $(\mathrm{P}>0.05)$.

\section{Discussion}

Based on the results of this study, it seems that social support among the school counselors is even more crucial than responsibility and unlike responsibility, which had no significant relationship with job procrastination. Social support can have a determining role in job procrastination since its existence improves confidence and self-competence and tests new experiences so that social support can be regarded among the strategies to modify the negative psychological pressures caused by the job. A supportive working environment is the most important factor to create job satisfaction for teachers and counselors because social support decreases the impact of psychological pressures and minimizes the effects of an unpleasant experience by increasing the proper understanding of stressful events. Besides, it creates mutual obligations because of which the individual feels that he is loved, cared and is valuable, these are directly related to reduced job procrastination and increased internal motivation to work.

As a result, the importance of providing such support on the part of educational officials is quite clear. Moreover, it was determined in the present research that basic psychological needs play a significant mediating role in the relationship between social support and job procrastination. In explaining this finding, it can be stated that according to the self-determination theory developed by Deci and Ryan, humans are motivated intrinsically and internally to engage in activities that are interesting to them, have an optimal challenge that is naturally pleasurable. This theory assumes that basic psychological needs satisfaction is essential for effective internalization of behaviors, psychological growth, motivation and wellbeing. For example, the need for self-command refers to the experience of a sense of choice, approval and free will in the beginning and completion of behavioral activities.

With respect to the raised concepts and the motivational forces created by the basic needs in the individuals, basic needs can be seen as an effective factor in reducing procrastination. Therefore, if the organization is a source that meets the need of employees, then a give and take relationship is formed in connection with the relationships between employees and the employer. In this context, the organizational support theory states that the creation of supportive relationships among employees leads to the formation of employees' overall view about the organization's support for themselves. In exchange for this support, they pay attention to the goals of the organization and their realization. In other words, when the organization considers the welfare of its employees, they mutually show more commitment and better performance in return for this consideration. Hence, it can be said that basic needs have a significant mediating role in the relationship between social support and job procrastination. So, it is better to put the satisfaction of these needs on the agenda of the relevant authorities. Given the small sample size and use of the correlation method which make causal conclusions impossible, further research is needed to confirm the accuracy of the results obtained in this study.

\section{Ethical Considerations}

\section{Compliance with ethical guideline}

This research was conducted with the approval of Research Council of Hakim Sabzrvari University and written informed consent was obtained from all participants. 


\section{Funding}

The research has done without any financial support.

\section{Conflict of interest}

The authors declare that they have no conflict of interest.

\section{Acknowledgements}

Hereby, we appreciate the authorities of Sabzevar Education Organization and the consultants who helped the researchers in this research. 


\title{
حمايت اجتماعى، مسئوليتيذيرى و اهمال كارى شغلى: نقش ميانجى ترانه ارضاى نيازهاى بنيادين

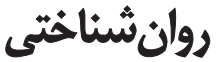

\author{
يروانه عمارلو'؛ "حسين شاره广
}

1- كارشناس ارشد، كروه روانشناسى، دانشكده علوم انسانى، واحد نيشابور، دانشعاه آزاد اسلامى، نيشابور، ايران.

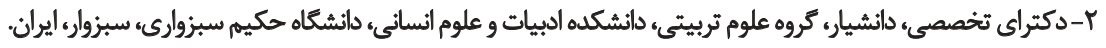

\begin{abstract}
حكبد

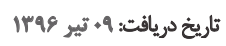

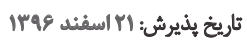

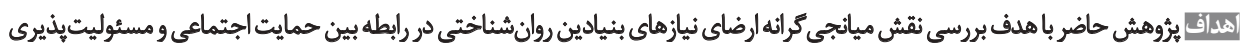

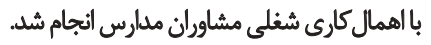

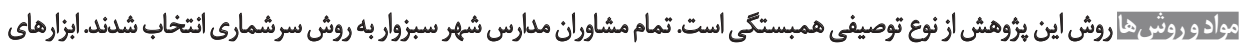

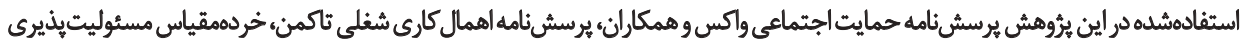

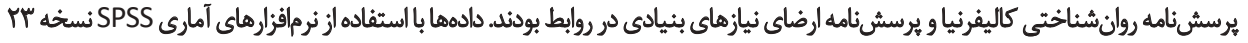
AMOS,

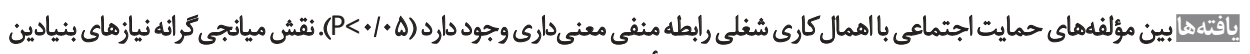

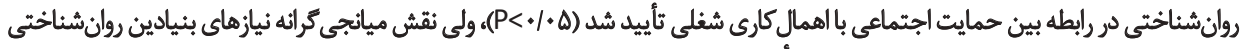

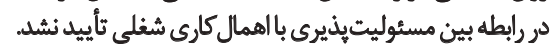

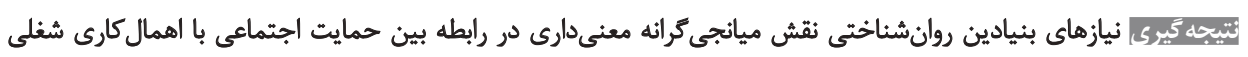
مشاوران ايفا مي كند.

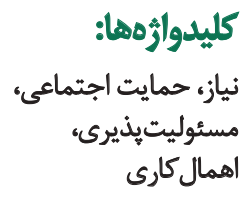

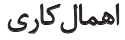

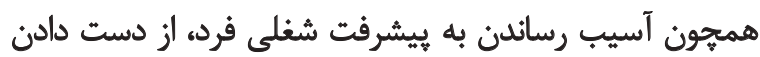

dale.

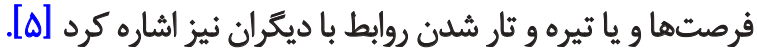

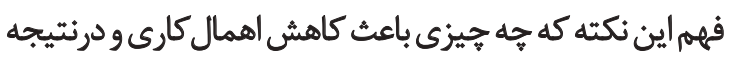

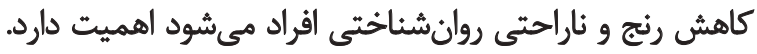

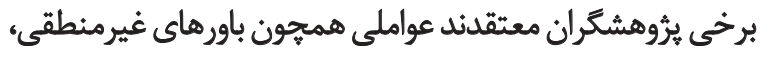

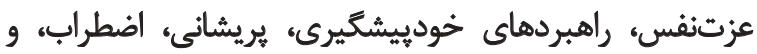

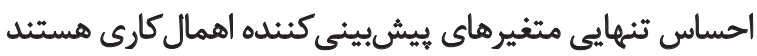

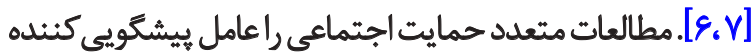

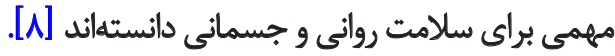

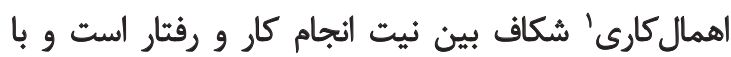

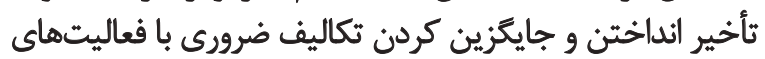

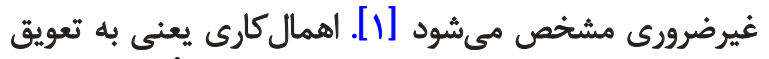

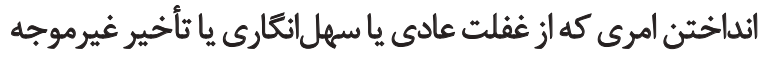

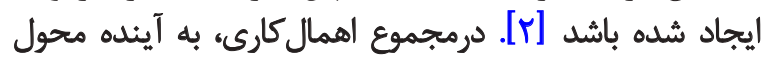

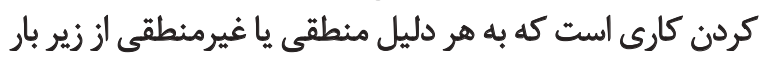

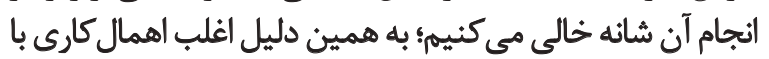

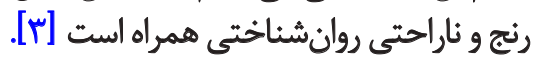

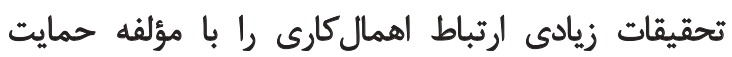

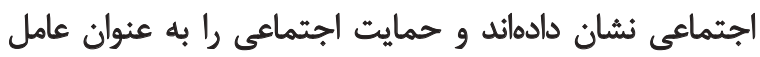

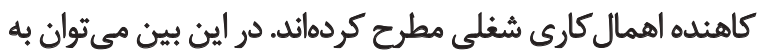

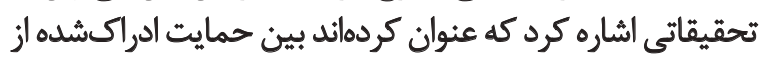

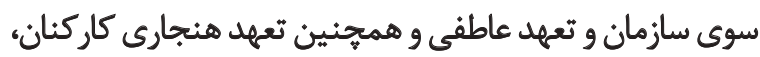

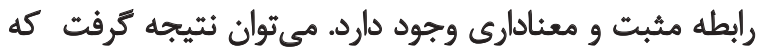

استيل [ [F] در خصوص بيامدهاي اهمال كارى بر افراد بيان

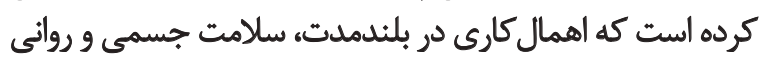

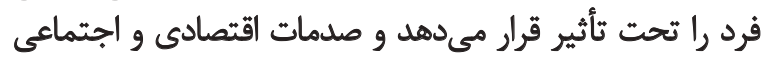

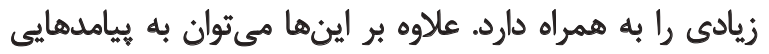

1. Procrastination

2. Steel

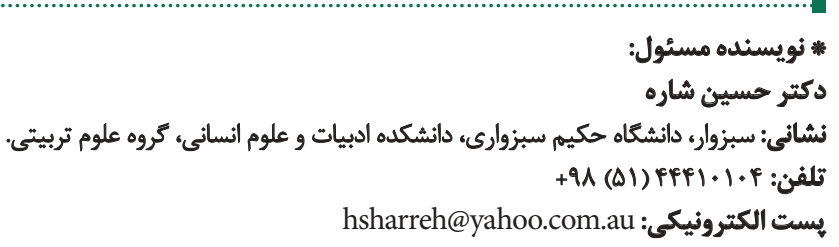


روانشناختى، انسجاميافتُكى و بهزيستى شخص فراهم و زيربناي

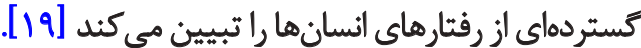
به عقيده دسى و رايان سه نياز اصلى انسانها خودمختاري،

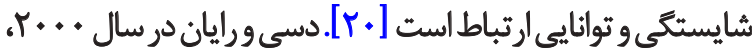

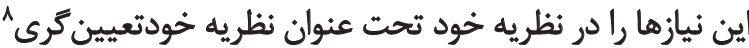

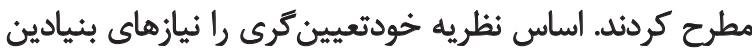

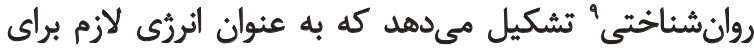

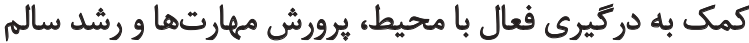

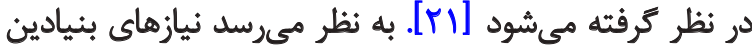

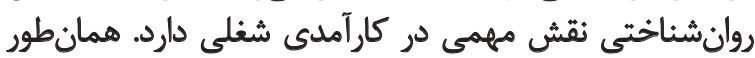

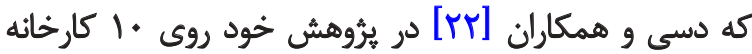

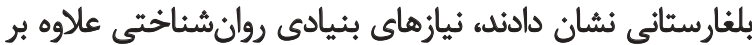

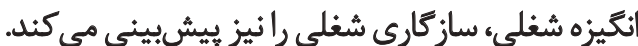

فتحى و همكاران [بr] در مطالعهاى رابطه بين نيازهاى

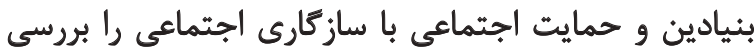

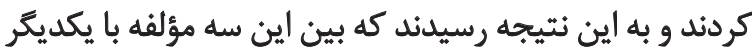

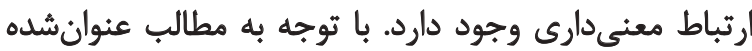

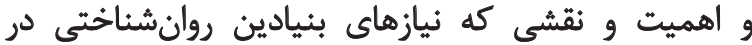

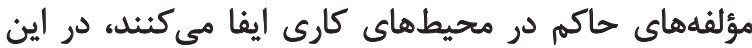

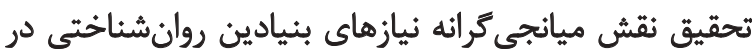

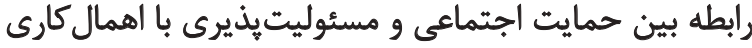

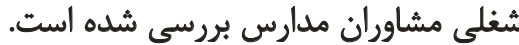

روش يُروهش حاضر، توصيفى و از نوع همبستى و به به طور

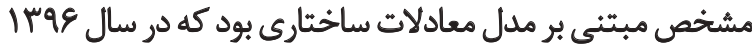

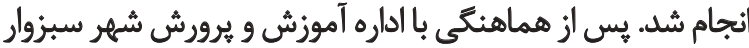

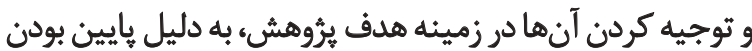

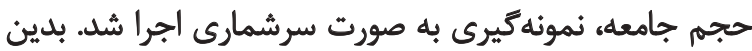

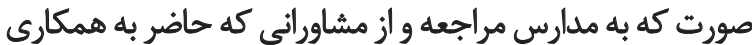

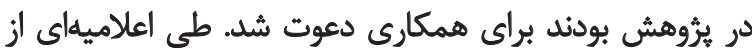

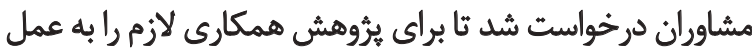

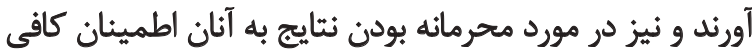

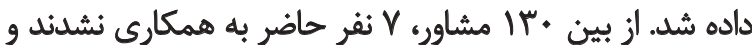

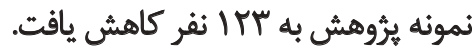

معيارهاي ورود آزمودنىها به يُروهش اين موارد بود:

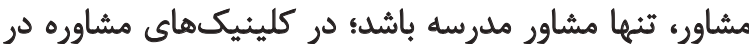

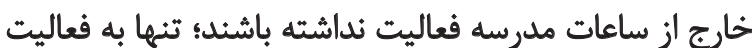

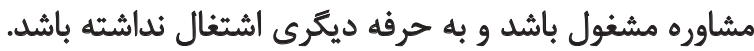

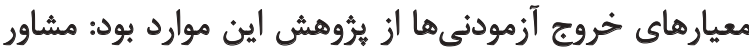

8. Theory of self-determination

9. Basic psychological needs
با وجود تعهد عاطفى و هنجارى كاركنان به كار، اهمال كارى

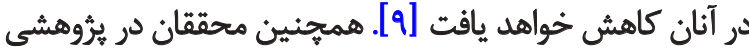

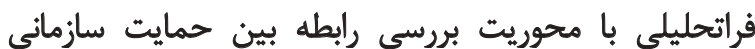

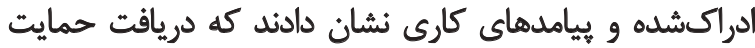

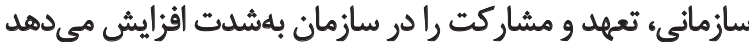

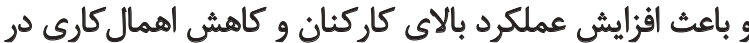

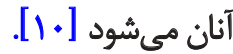

منظور از حمايت اجتماعى، كمكهاى عاطفى، ابزارى و

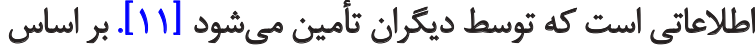

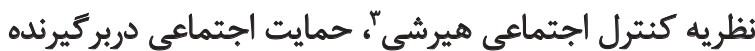

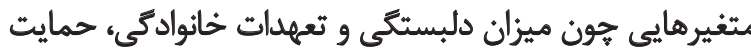

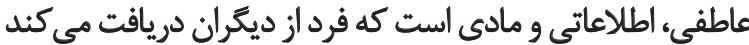

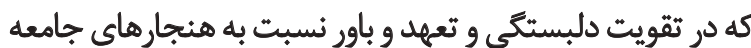

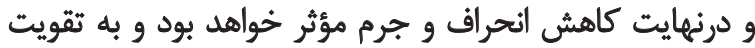

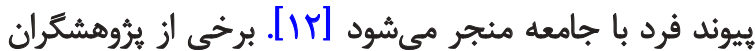

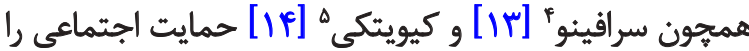

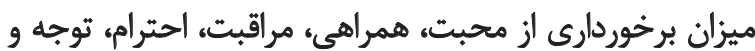

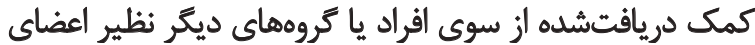

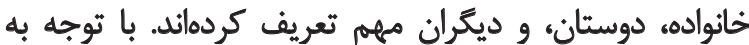

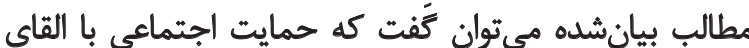

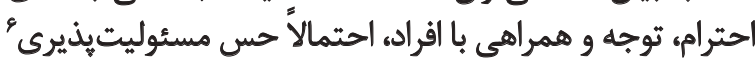
متقابل رادر آنان تقويت مي كمند.

مى توان فرض كرد كه عامل مسئوليث يذيرى در افراد يكى از

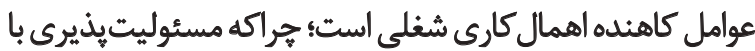
تقويت صفاتى جون تدبير، اعمال متفكرانه، سازماندها

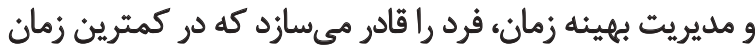

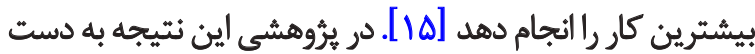

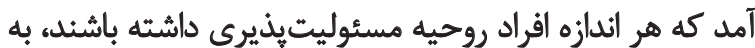

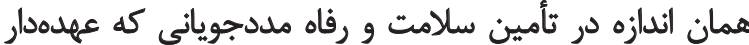

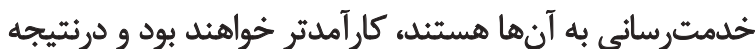

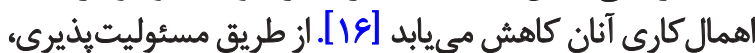

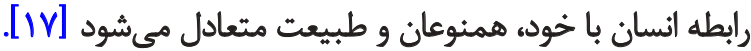

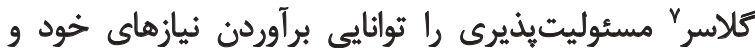

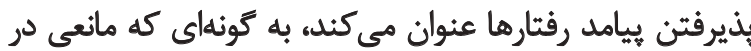

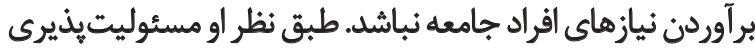

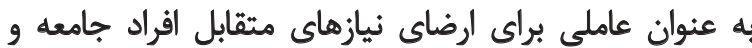

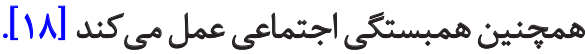

نيازهاى بنيادين روانشناختى، شرايط لازم را براى رشد

3. Hirschi

4. Sarafino

5. Çivitci

6. Responsibility

7. Glasser 
به بالا باشده نشاندهنده اهمال كارى زياد در فرد است. تاكمن

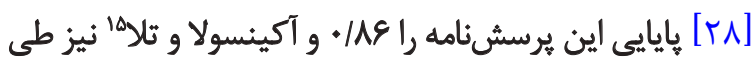

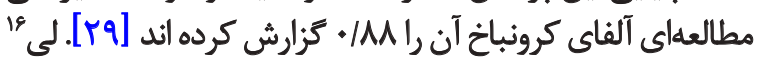

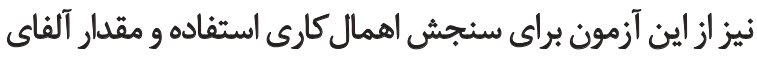

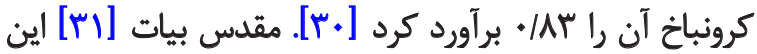

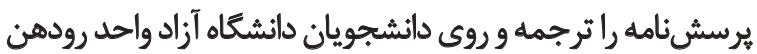

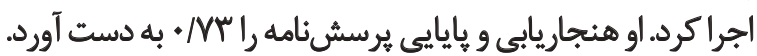

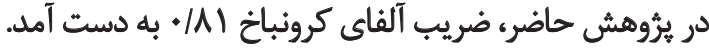

\section{"يرسشنامه نيازهاى بنيادى روانشناختى تارديا وهمكاران"}

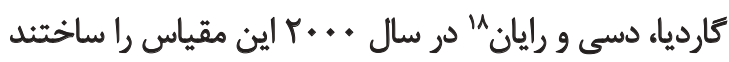

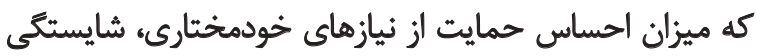

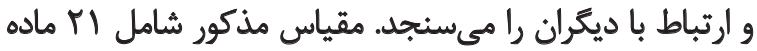

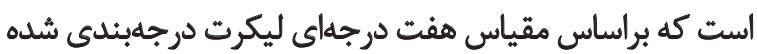

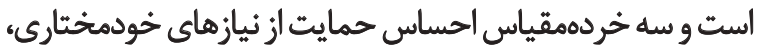

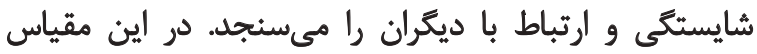

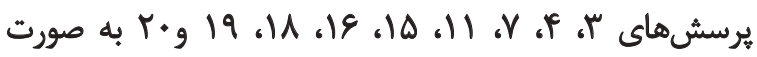

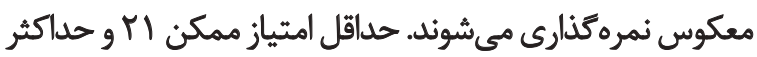

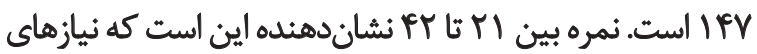

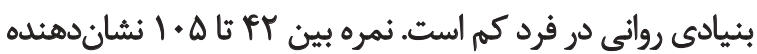

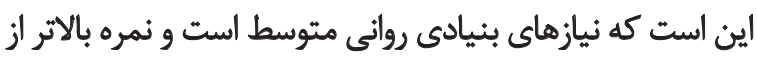

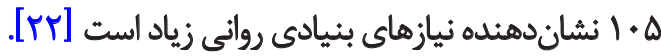

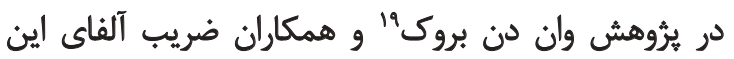

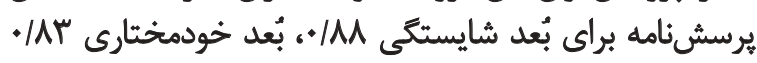

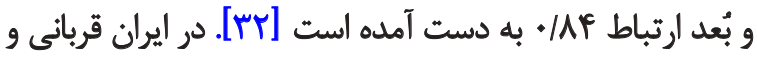

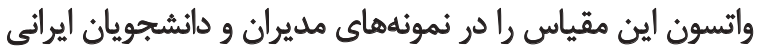

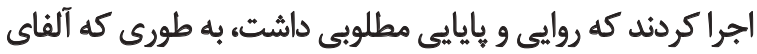

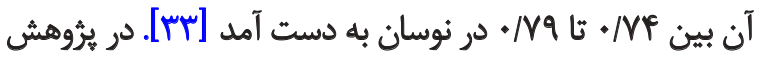

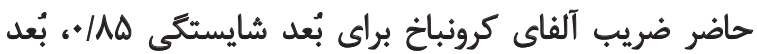

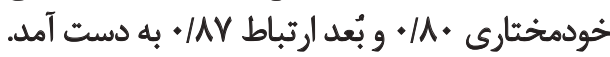

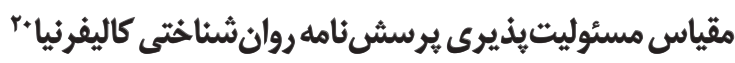

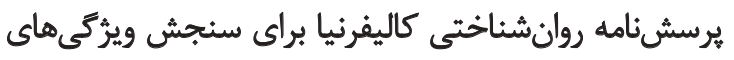

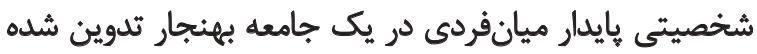

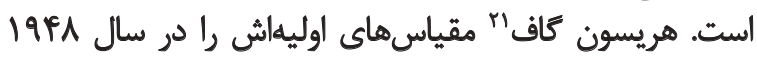

\section{Tuckman}

15. Akinsola \& Tella

16. Lee

17. Basic Needs Satisfaction in Relationships Scale

18. Guardia, Deci and Ryan

19. Van den Broeck

20. California Personality Inventory (CPI)

21. Harrison Gough
مشغول به تحصيل باشد؛ به بيمارىهاى روانشناختي بارز

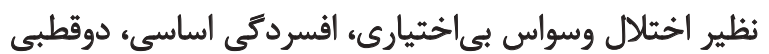

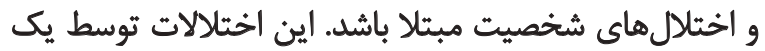

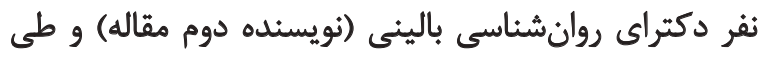

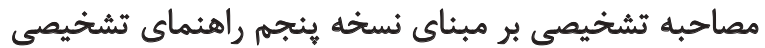

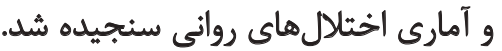

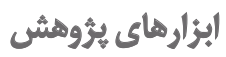

مقياس حمايت اجتماعى واكس و همكاران"

واكس" و وركاران يرسش إنامه حمايت اجتماعي را تهيه

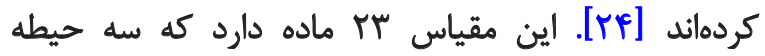

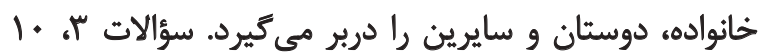

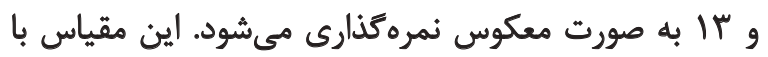

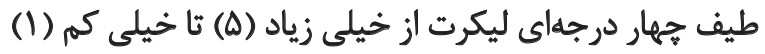

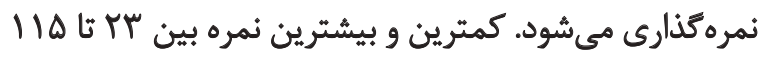

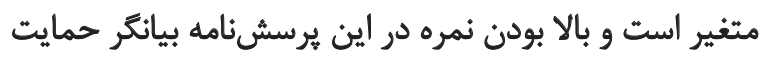

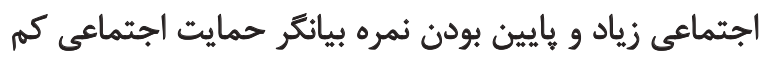

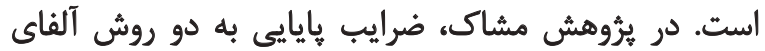

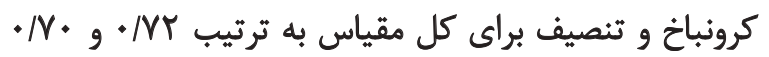

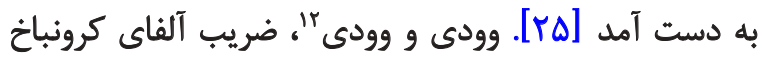

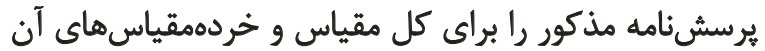

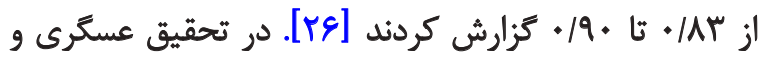

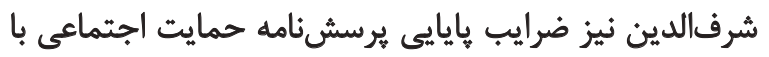

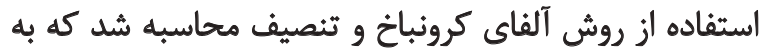

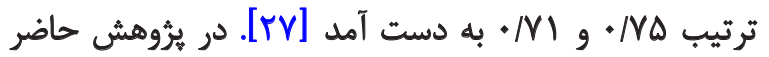

$$
\text { ضريب آلفاى كرونباخ /VA • • به دست دست آمد. }
$$

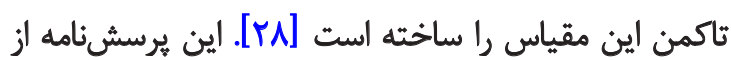

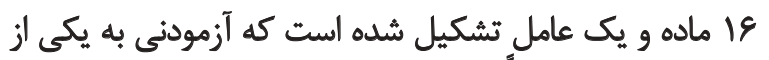

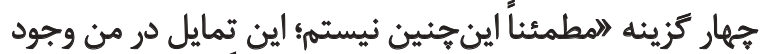

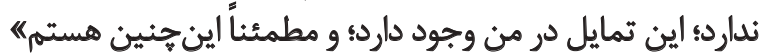

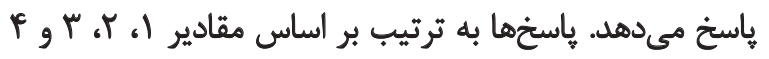

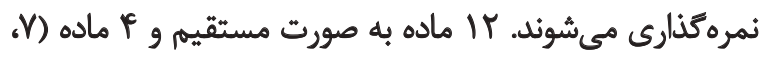

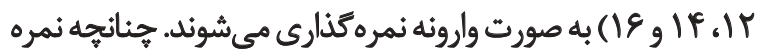

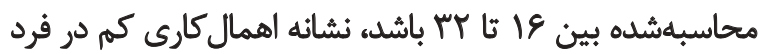

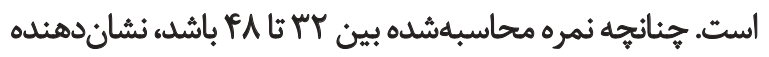

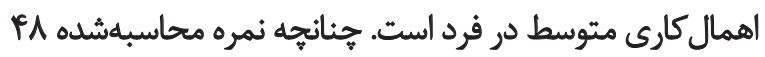

10. Social support

11. Vaux

12. Woody

13. Tuckman Procrastination Scale 


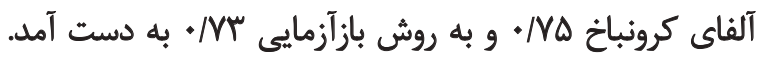

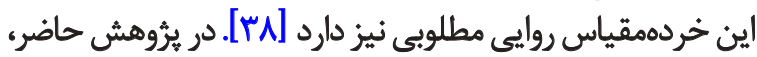

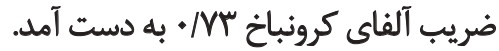

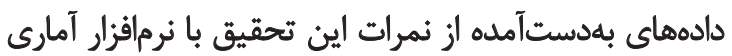

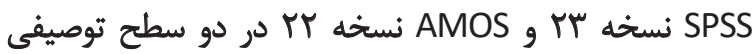

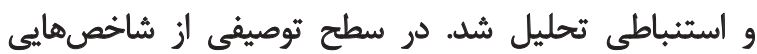

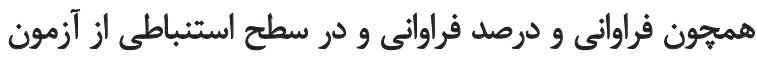

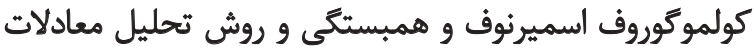
ساختارى استفاده شد. - سورف

\section{ياقتههها}

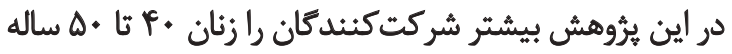

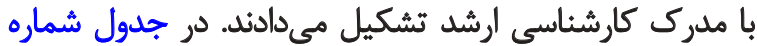

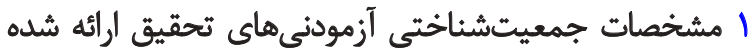

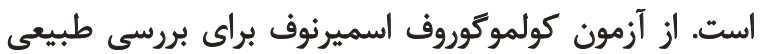

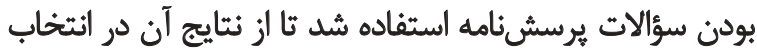

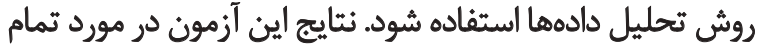

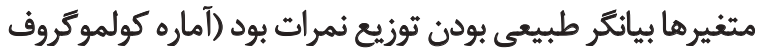

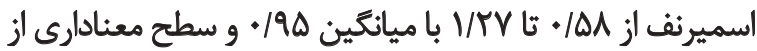

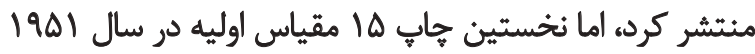

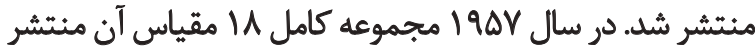

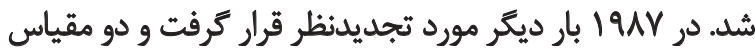

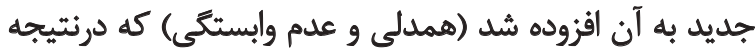

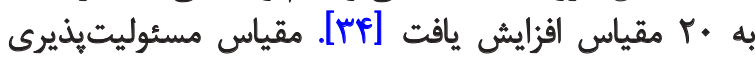

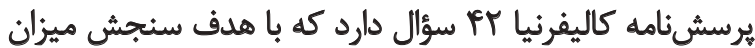

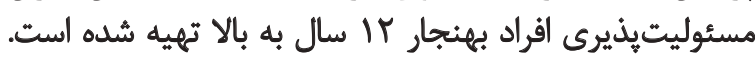

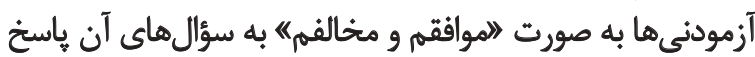

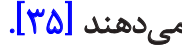

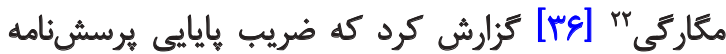

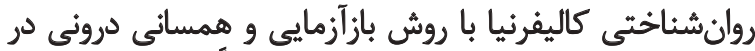

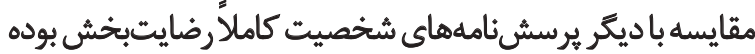

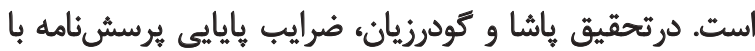

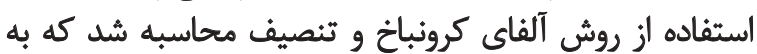

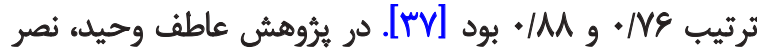

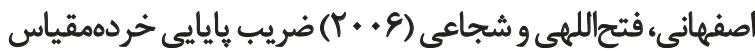

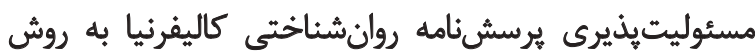

22. Megargee

جدول ا. ويُّكى هاي جمعيتشئاختى نمونه مطالعه

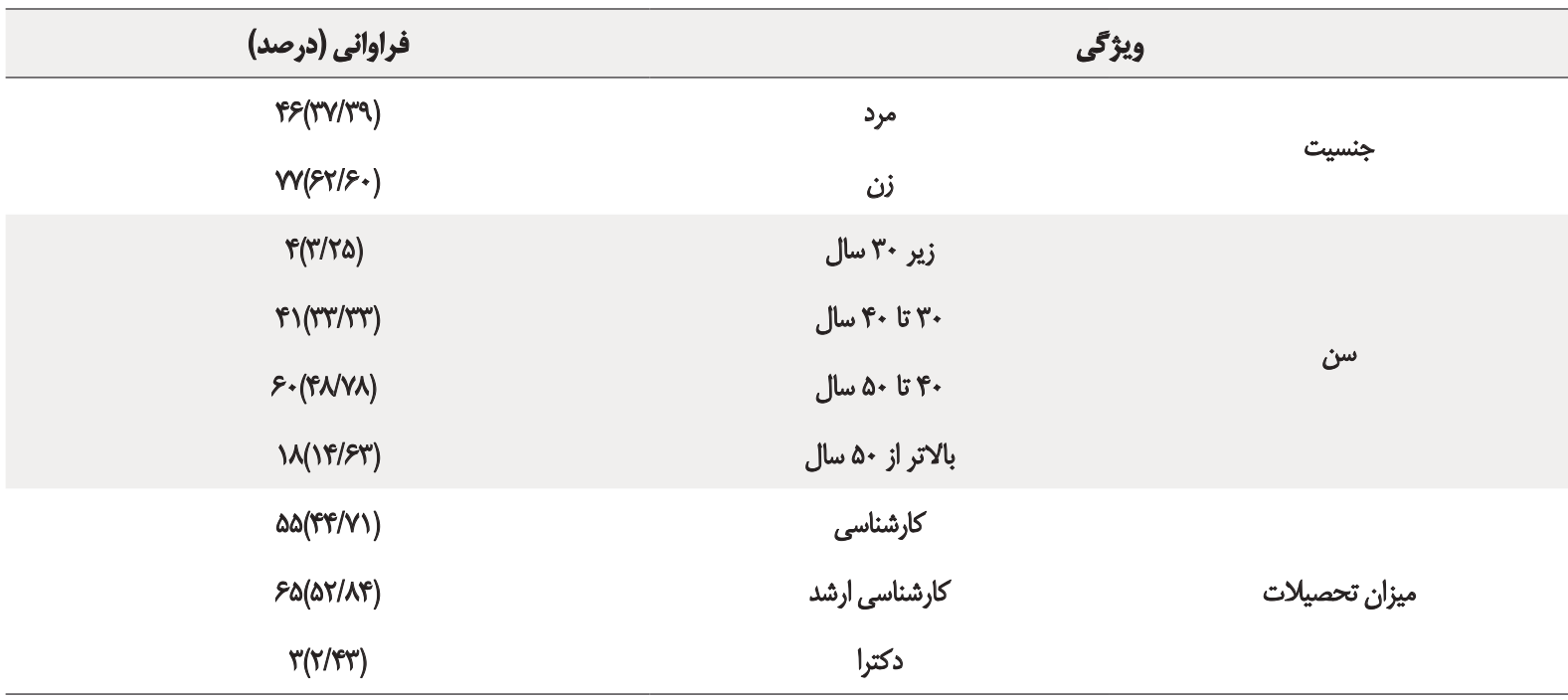

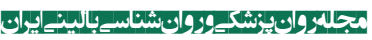

جدول ז. همبستكي بين مؤلفههاى بيشبين و ميانجي با مؤلفه ملاك

\begin{tabular}{|c|c|c|c|c|c|c|c|}
\hline الرتباطيان بان & شايسيّكى & نياز خودمخثتارى & مسئوليتيذيرى & ديكرانيت & دوستان & خانواده & مؤلفهها \\
\hline$-+/ / \mu$ & $-+/ T \Delta$ & $-4|\pi|$ & 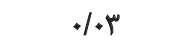 & $-t / 4$ & $-+/ M E$ & 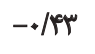 & همبستكى (اهمال كارى شغلى) \\
\hline $.1+1$ & $+\infty 1$ & 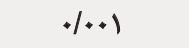 & $\cdot M T$ & $.10+1$ & 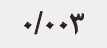 & $+\infty 1$ & سطح معنى دارى \\
\hline
\end{tabular}




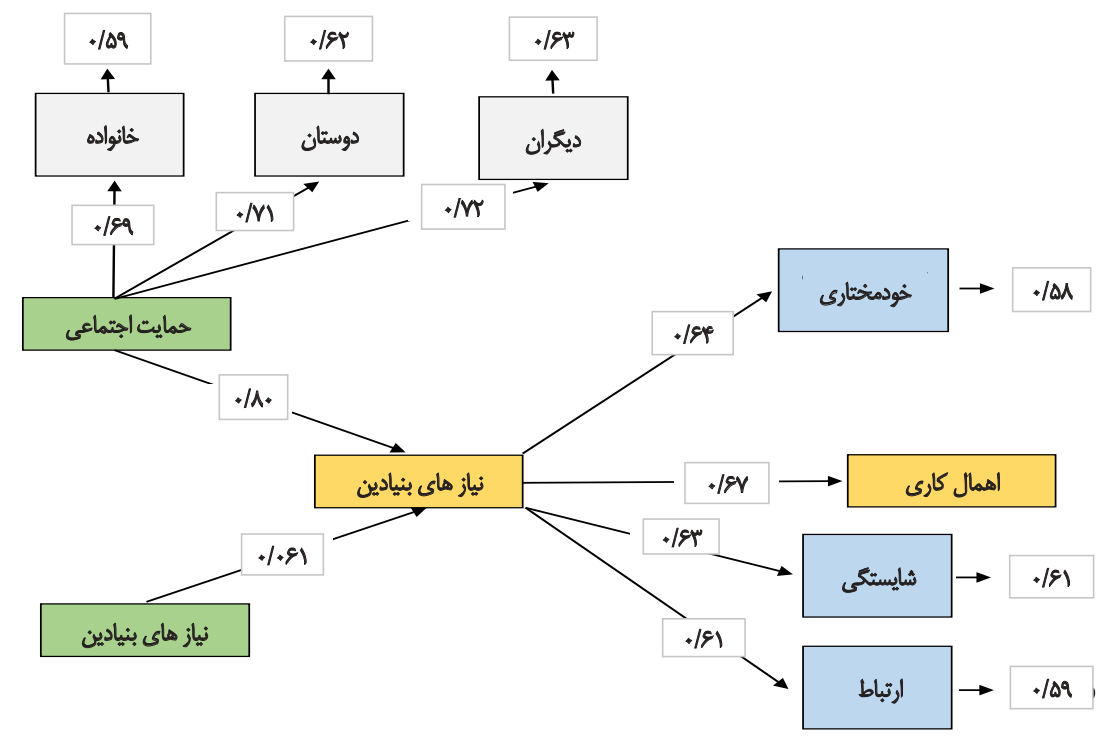

ساختاري بررسى شد.در تصوير شماره إضريب تخمين استاندارد

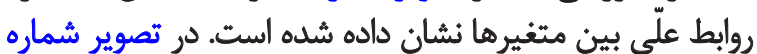

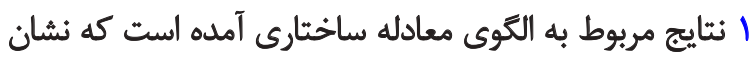

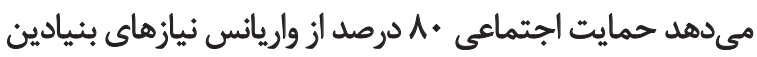

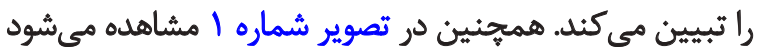

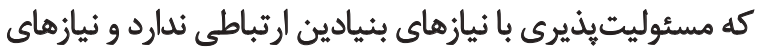

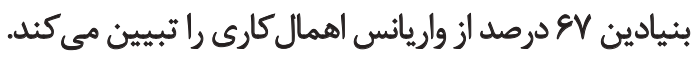
همانطور كه در تصوير شماره Y مشاهده مي شود به منظور

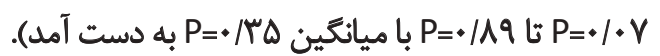

جدول شماره Y همبستى بين مؤلفه هاى تحقيق را با يكديكر

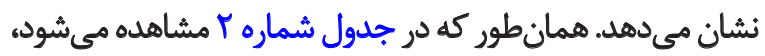

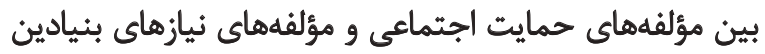

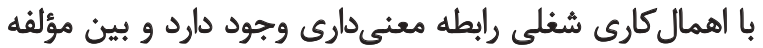

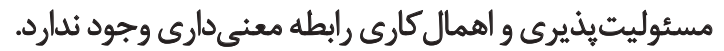
براى آزمون مدل مفهومي تحقيق بر اساس فرضيههاى تحقيق،

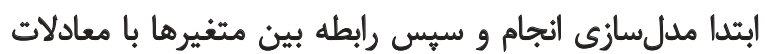

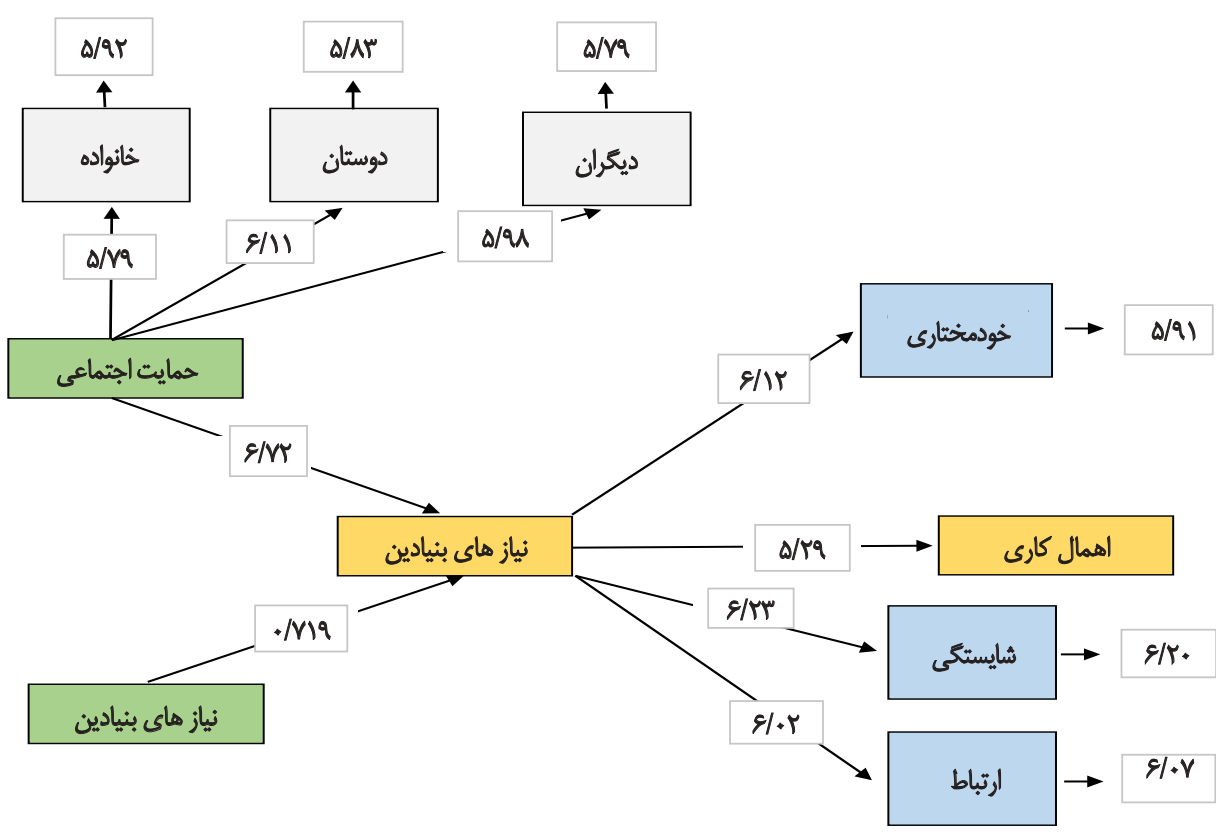

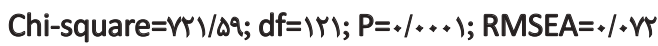


جدول "َ. برازش مدل ساختارى تحقيق

\begin{tabular}{|c|c|c|c|c|}
\hline تثيجه & معيار ها در تحقيث حاضر & معيارهاى مدل برازئده & نام أزمون & رديف \\
\hline مدل برازنده & $+1+\infty 1$ & معنادار باشد. & $X^{r}$ & 1 \\
\hline مل برازئل & .1 .11 & هر جيه به صفر نزوديكتر باشد. & RMR & r \\
\hline ملل برازئده & .19 & بين صفر و يك، برابر يا بزركتر از ه/. & GFI & $r$ \\
\hline 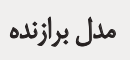 &.$/ 94$ & بين صفر و يك، برابر يا بزركتر از ه/. & AGFI & f \\
\hline ملل برازئده & $.1 \cdot v$ & كوجهتر از $1 /$ • باشد. & RMSEA & $\Delta$ \\
\hline مل بل مازئله & .19 & بايد بزركتر از ه/ • بانش. & $\mathrm{NFI}$ & 8 \\
\hline مل بل برازنلده & +191 & بايد بزركثر از ه/• باشش. & $\mathrm{CFI}$ & $\checkmark$ \\
\hline
\end{tabular}

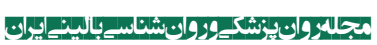

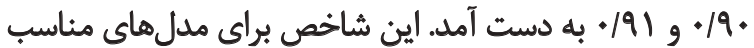

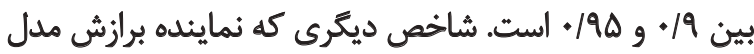

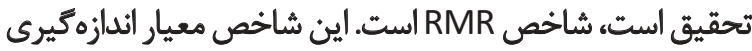

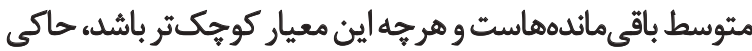

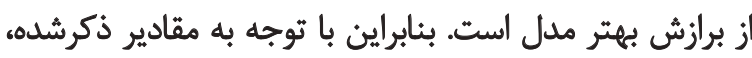

$$
\text { مدل برازش مناسبى دارد. }
$$

جدول شماره f بارهاى عاملى استانداردنشده (b)، بار عاملى

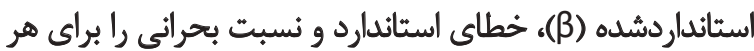

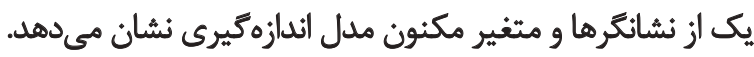

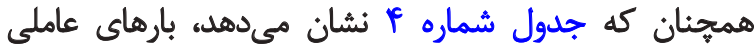

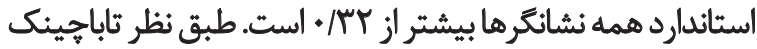

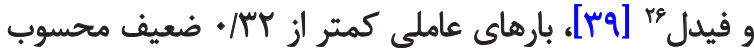

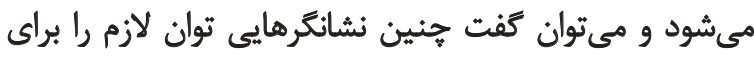

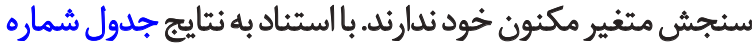

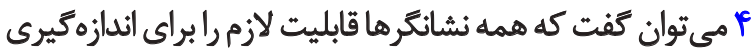

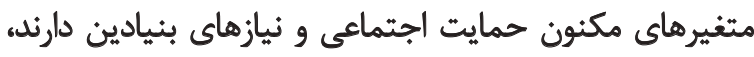

26. Tabachink \& Fidel t نشان دادن معنادارى هر كدام از متغيرهاى مدل از آماره

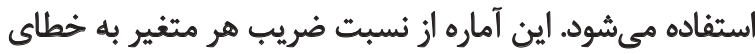

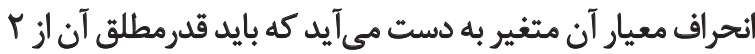

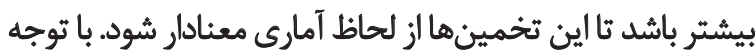

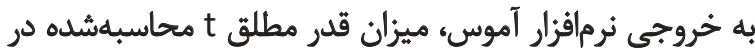

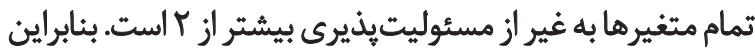

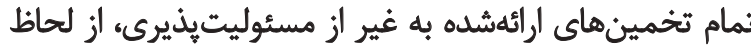

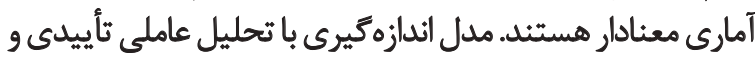

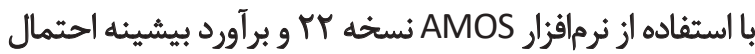

$$
\text { (ML) }
$$

در اين تحقيق مجذور كاي معنادار است. همجنين ريشه

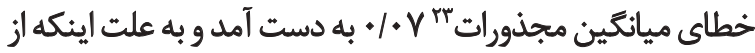

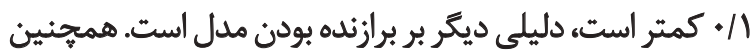

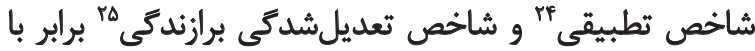

23. Root Mean Square Error of Approximation (RMSEA)

24. Comparative fit index (CFI)

25. Adjusted Goodness of Fit Index (AGFI)

\begin{tabular}{|c|c|c|c|c|}
\hline نسبتبحرانى & خطاى استاندارد & بارعاملى استاندارد (B) & بار عاملى استاندار دنششده (b) & متغيرهاى مكنون - نشانكر \\
\hline & &.$/ \Delta q$ & 1 & حمايت اجتماعى، حمايت خانواده \\
\hline $9 / 1$ & $\% 11 \mathrm{~A}$ &.$/ 4 T$ & $1 / N$ & تمايت اجتماعى، حمايت دوستان \\
\hline \multirow[t]{2}{*}{$\Delta / u$} & $\pi / 14$ & . & $1 / \%$ & حمايتاجتماعى، حمايت ديكران \\
\hline & &.$/ A \mathrm{~A}$ & 1 & نيازهاى بنيادين،خومثختارى \\
\hline $9 / \pi$ & $\%$ & .191 & $1 / 41$ & نيازهاى بنيادين،شايستكى \\
\hline ध.r &.$/ 18$ &.$/ 109$ & $1 / 49$ & نيازهاي بنيادين، ارتباطا \\
\hline
\end{tabular}

جدول F. متغيرهاى مدل اندازهكيرى يُزوهش در تحليل عاملى تأييدى 
جدول هـ جدول كلى آزمون فرضيات تحقيق بر اساس مدل معادله ساختار تحقيق

\begin{tabular}{|c|c|c|c|c|}
\hline نتيجه & $\mathbf{T}$ & ضريب & ادعاى فرضيه تحقيث & شماره \\
\hline تأيبيد & $g / N r$ & $\cdot / 1$ & حمايت اجتماعى -ـنيازهاى بنيادين & 1 \\
\hline د) & $\cdot M$ & .1 .8 & مسئوليت يذيرى = ثيازهاي بنيادين & r \\
\hline ثأييد & $\Delta / R q$ & $-18 \mathrm{~V}$ & ثيازهاى بنيادين تـ اهمال كارى شغلى & $r$ \\
\hline
\end{tabular}

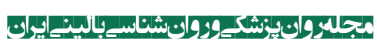

بركمن و سيم" [Ff] به عنوان نظريه يردازان ايده تأثير حمايت

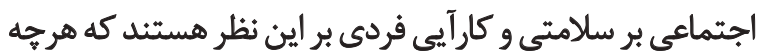

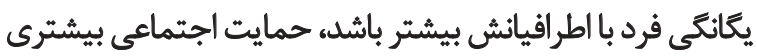

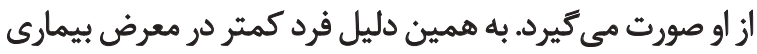

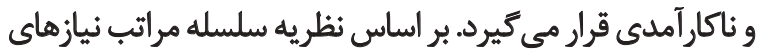

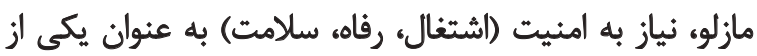

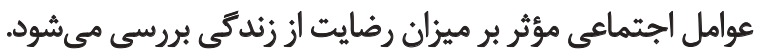

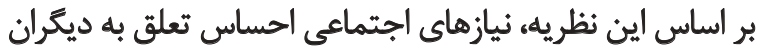

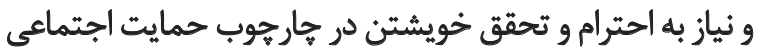

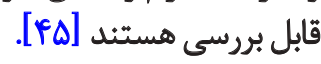
وجود حمايت اجتماعى به اطمينان و خودشايستكى ها براي براي

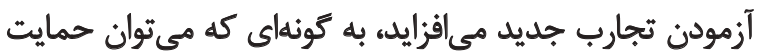

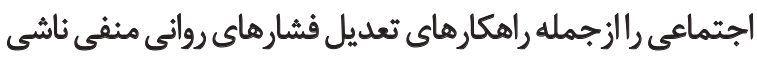

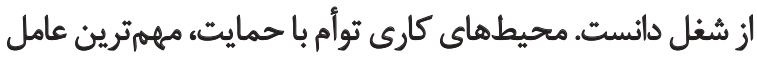

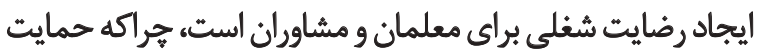

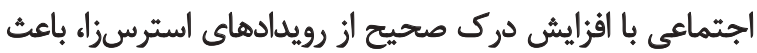

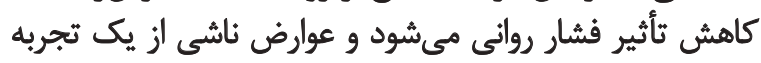

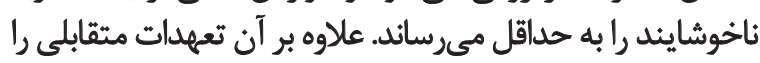

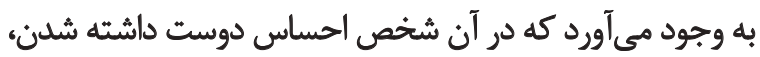

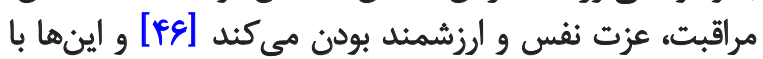

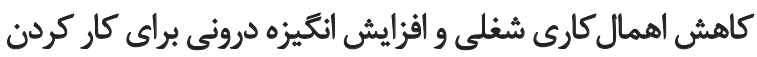

$$
\text { ارتباط مستقيمى دارد. }
$$

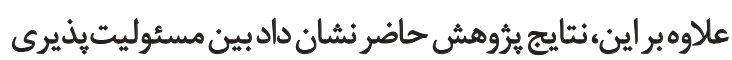

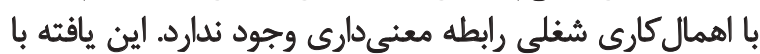

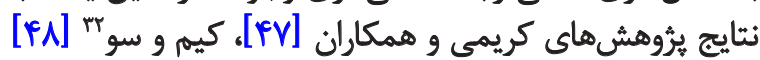

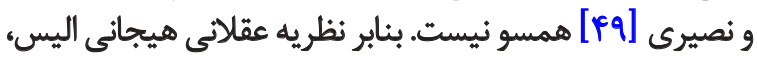

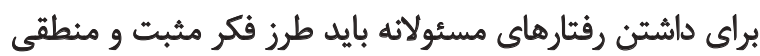

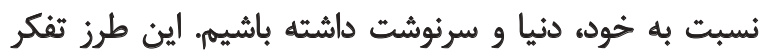

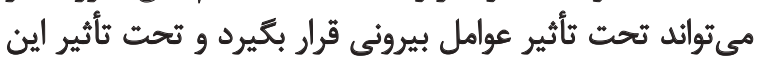

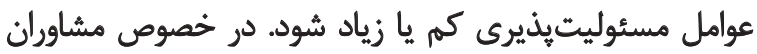

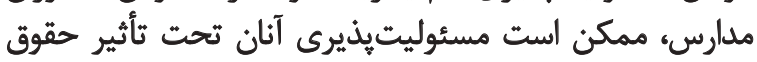

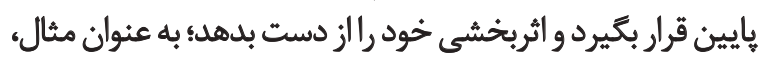

31. Berekman and Sim

32. Kim and Seo

$$
\text { درئتيجه تحليل معادلات ساختارى امكانيذير است. }
$$

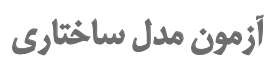

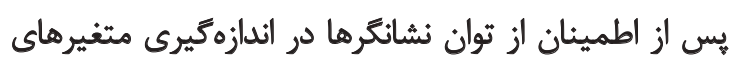

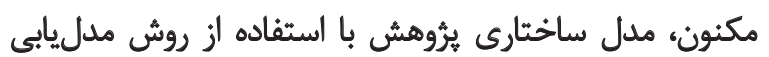

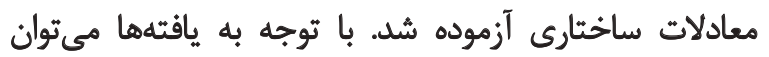

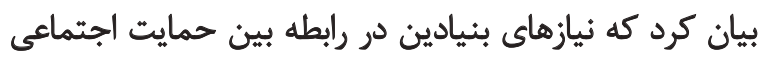

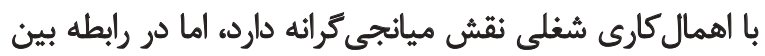
مسئوليتيذيرى با اهمال كارى شغلى نقش مياني ميانجى كرانه ندارد.

ث

يثروهش حاضر با هدف بررسى نقش ميانجى كرانه نيازهاى

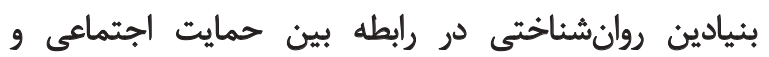

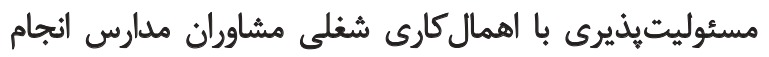

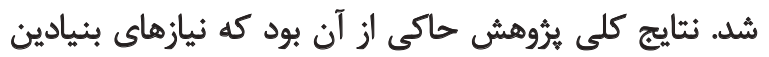

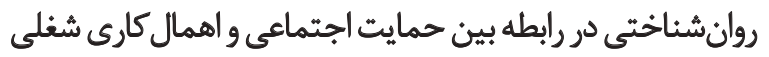

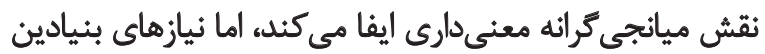

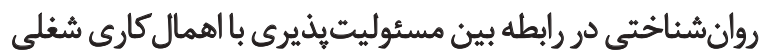

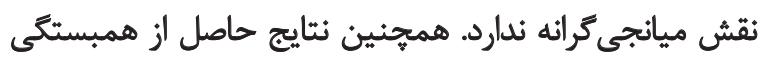

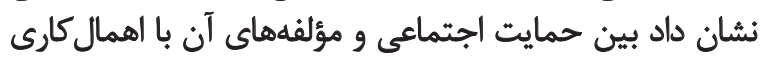

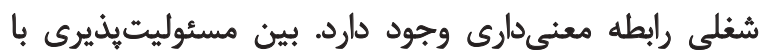
اهمال كارى شغلى رابطه معنى دارى يافت نشد.

نتايج يُوهش حاضر مبنى بر وجود رابطه بين حمايت اجتماعى بإي

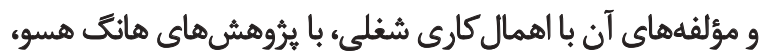

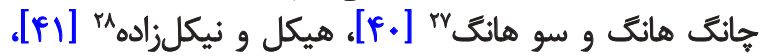

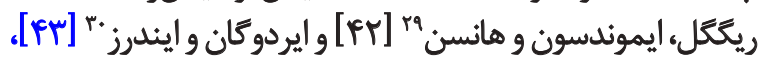

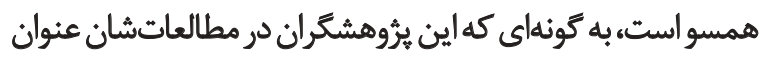

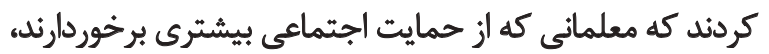

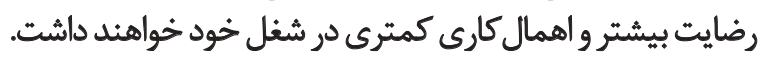

27. Hung Hsu, Chung Huang, and Sue Huang 28. Hikel and Nekelzadeh

29. Riggle, Edmondson, and Hansen 30. Erdogan and Endres 
در خصوص نداشتن نقش ميانجيةًرائه نيازهاى بنيادين در

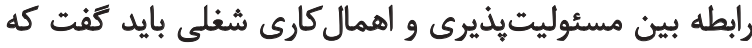

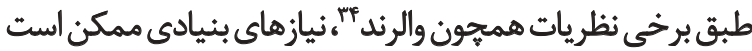

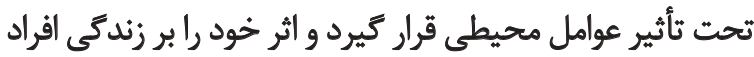

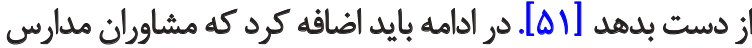

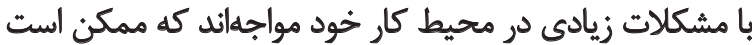

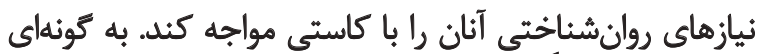

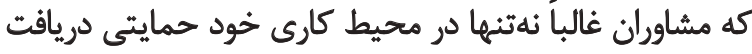

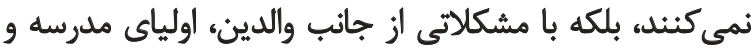

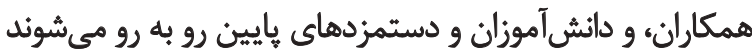

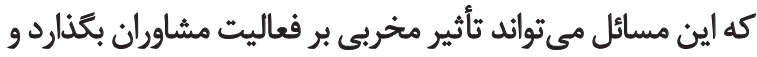

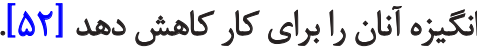

در عصرى كه سازمانها و محيطهاي كارى هر روز با جالشها

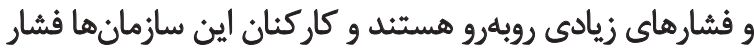

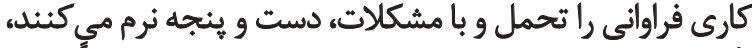

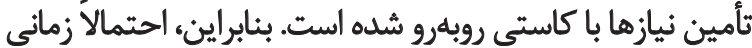

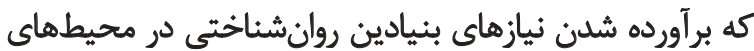

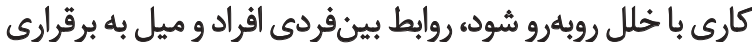

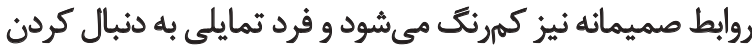

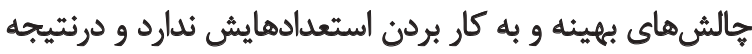

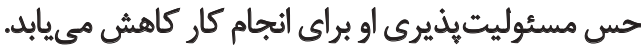
حجم نمونه كم محدوديت اصلى يثروهش حاضر است و استفاده

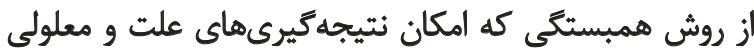

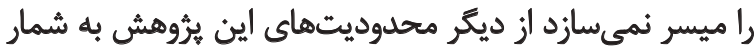

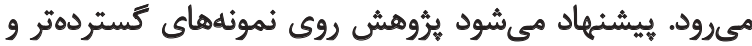

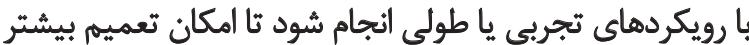

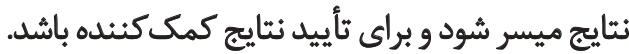

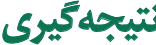

نتايجيثروهش حاضرنشان دادبين حمايت اجتماعى بالهمال كارى

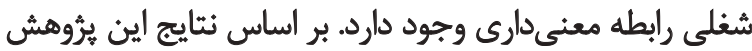

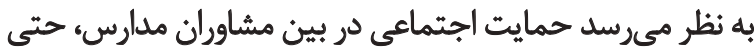

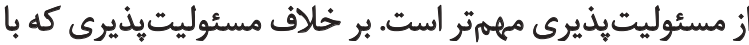

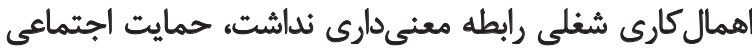

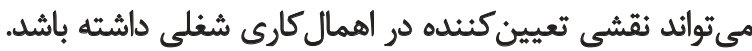

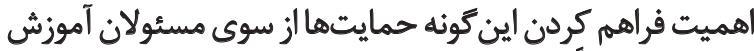

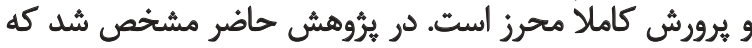

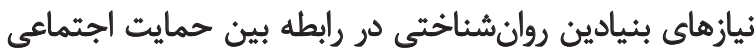

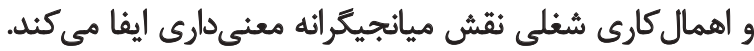

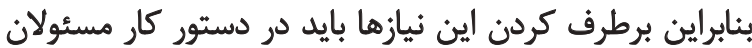

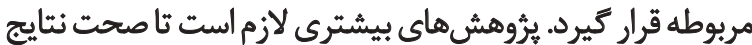

افرادى كه زمينه بيشترى براي اهمالورزى دارنله، در اثر ياداداش

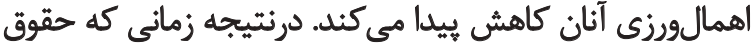

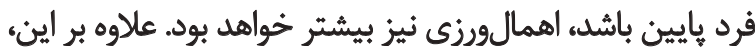

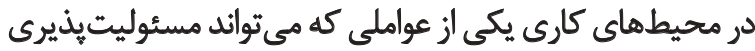

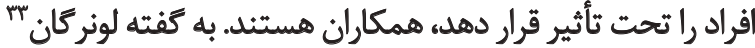

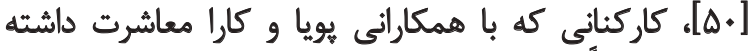

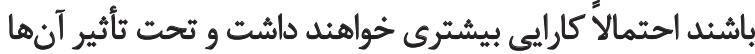

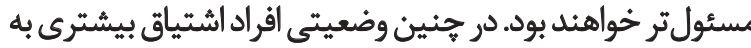

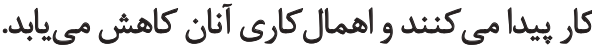

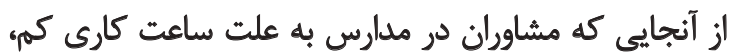

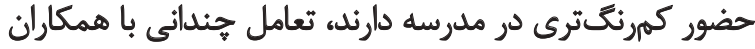

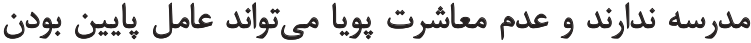

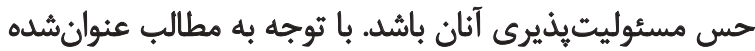

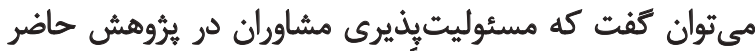

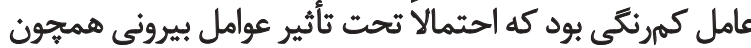

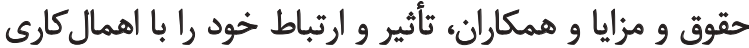

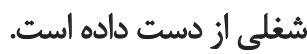

در تبيين نتايج حاضر مبنى بر نقش ميانجى

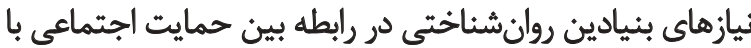

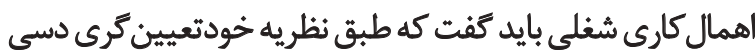

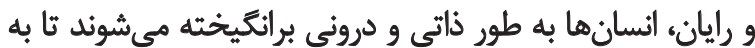

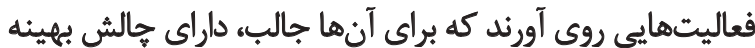

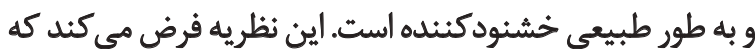

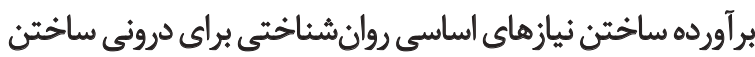

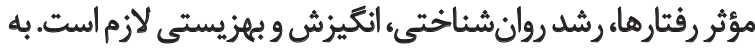

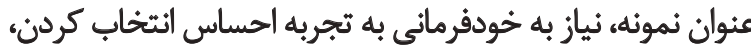

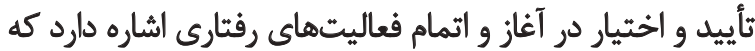

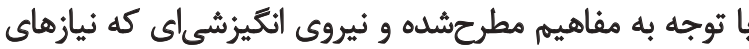

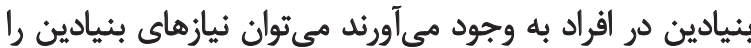

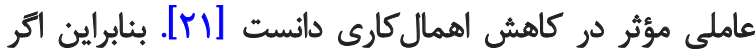

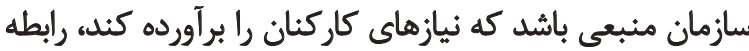

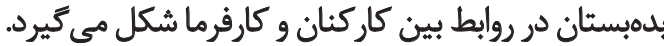
در همين راستا نظريه حمايت سازمانى بيان ميكى دند كه

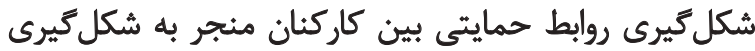

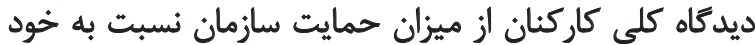

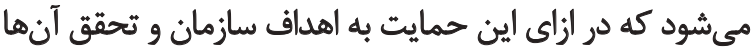

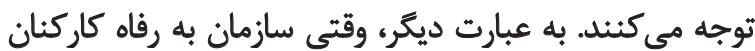

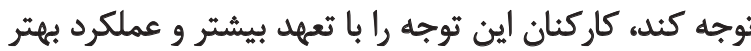

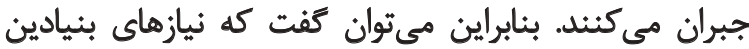

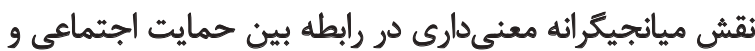
اهمال كارى شغلى دارد. 
بلهدستآمده در اين يرُوهش را روى نمونههاي ديكر تأييد شود.

$$
\text { سياسكزازإى }
$$

اين مقاله از ياياننامه كارشناسى ارشد نويسنده اول در دانشكده

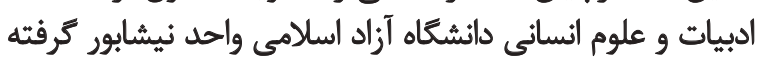

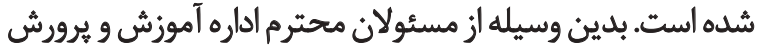

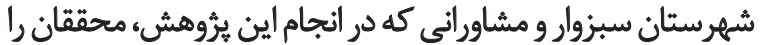
يارى كردند تقدير و تشكر به عمل مئ كنيه.

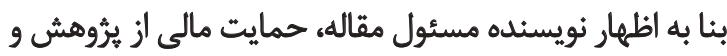
تعارض منافع وجود نداشته است. 


\section{References}

[1] Schouwenburg HC. Procrastination in academic settings: General Introduction. In Schouwenburg HC, Lay CH, Pychyl TA, Ferrari JR, editors. Counseling the procrastinator in academic settings. Washington, D.C.: American Psychological Association; 2004.

[2] Badri Gargari R, Fathiazar E, Mohammadi N. [The relationship between self-forgiveness and positive and negative emotions with academic procrastination in third grade high school students (Persian)]. Journal of School Psychology. 2014; 3(1):6-21.

[3] Wohl MJ, Pychyl TA, Bennett SH. I forgive myself, now I can study: How self-forgiveness for procrastinating can reduce future procrastination. Personality and Individual Differences. 2010; 48(7):803-8. [DOI:10.1016/j.paid.2010.01.029]

[4] Steel P. The nature of procrastination: A meta-analytic and theoretical review of quintessential self-regulatory failure. Psychological Bulletin. 2013; 133(1): 65-94. [DOI:10.1037/0033-2909.133.1.65] [PMID]

[5] Ahmadi Z. [The role of goal orientation and motivational beliefs in academic procrastination among third year high school students in Tabriz (Persian)] [MSc. Dissertation]. Tabriz: Azarbaijan Shahid Madani University; 2011.

[6] Öksüz, Y., \& Güven, E. The relationship between psychological resilience and procrastination levels of teacher candidates. Social and Behavioral Sciences. 2014; 11(6):3189-93. [DOI:10.1016/j.sbb spro.2014.01.732

[7] de la Fuente J, Sander P, Martínez-Vicente JM, Vera M, Garzón A, Fadda S. Combined effect of levels in personal self-regulation and regulatory teaching on meta-cognitive, on meta-motivation$\mathrm{al}$, and on academic achievement variables in undergraduate students. Frontiers in Psychology. 2017; 8:232. [DOI:10.3389/fpsyg.2017.00232]

[8] Miller LM. The interrelationship between social support and post-trauma symptoms in the aftermath of a severe motor vehicle accident. Buffalo, New York: State University of New York at Buffalo; 2007.

[9] Taleghani GhR, Divandari A, Shir Mohammadi M. [The effictiveness of perceived support as organizational on employee commitment and organizational performance: A study in branches of Bank Mellat in Tehran (Persian)]. Iranian Journal of Management Sciences. 2009; 4(16):1-25.

[10] Riggle RJ, Edmondson DR, Hansen JD. Meta analysis of the relationship between perceived organizational support and job outcomes, 20 years of research. Journal of Business Research. 2009; 62(10): 1027-30. [DOI:10.1016/j.jbusres.2008.05.003]

[11] Gerard JM, Landry Meyer L, Roe JG. Grandparents raising grandchildren the role of social support in coping with caregiving challenges. International Journal of Aging \& Human Development. 2006; 62(4): 359-83. [DOI:10.2190/3796-DMB2-546Q-Y4AQ] [PMID]

[12] Davis NJ, Stasz C. Social control of deviance: A critical perspective.New York: McGraw-Hill Publishing Company; 1990.

[13] Sarafino EP, Smith TW. Health psychology: Biopsychosocial interactions.NewYork: John Wiley \& Sons; 2014 Jan 13.

[14] Çivitci A. The moderating role of positive and negative affect on the relationship between perceived social support and stress in college students. Educational Sciences: Theory \& Practise. 2015; 15(3): 25-53. [DOI:10.12738/estp.2015.3.2553]
[15] Gharabagi H. [The relationships between traits character and job satisfaction among Iran tractor plant employees (Persian) [MSc. Dissertation]. Tabriz: Tabriz University of Medical Sciences; 2003

[16] Beikzad J, Hoseinpour A, Hejazi Bavil M. [A survey on the relationship between responsibility and job satisfaction of nurses working in teaching hospitals affiliated with Tabriz University of Medical Sciences (Persian)]. Journal of Hospital. 2014; 13(1):53-60

[17] Soheili H. [Examine the relationship between self-actualization responsibility, locus of control and creativity among graduate students in Ahvaz city (Persian)] [MSc. Dissertation]. Ahvaz: Islamic Azad University of Ahvaz; 2008.

[18] Glasser W. Management without coercion (selection theory in human resource management [Shabi A, Persian trans.]. Tehran: SayehSokhan Publication; 2011.

[19] Ghasemipour Y, Bahrami H, Abaspour S, Poursharifi H. [The effectiveness of motivational interviewing on satisfying basic psychological needs among patients under Coronary Artery Bypass Grafting (CABG) (Persian)]. Journal of Clinical Psychology. 2011; 3(2):43-54.

[20] Deci E, Ryan RM. Intrinsic motivation and self-determination in human behavior. Berlin: Springer Science \& Business Media; 19851. [DOI:10.1007/978-1-4899-2271-7] [PMID]

[21] Deci EL, Ryan RM. Levels of analysis, regnant causes of behavior and well- being: The role of psychological needs. Psychological Inquiry. 2011; 22; 17-22. [DOI:10.1080/1047840X.2011.545978]

[22] Deci EL, Ryan RM, Gagne M, Leone DR, Usunov J, Kornazheva BP. Need satisfaction, motivation, and well-being in the work organizations of a former eastern bloc country: A cross-ultural study of self-determination. Journal of Personality and Social Psychology. 2000; 27(8): 930. [DOI:10.1177/0146167201278002]

[23] Fathi A, Rezapoor Y, Yaghooti Azar Sh. [The relationship between basic needs and social support with social adjustment among male and female students (Persian)]. The Journal of Social Studies. 2010; 25: 133-146.

[24] Vaux A, Phillips J, Holly L, Thomson B, Williams D, Stewart D. The Social Support Appraisals (SSA) scale: Studies of reliability and validity. American Journal of Community Psychology . 1986; 14(2):195-218.[DOI:10.1007/BF00911821]

[25] Meshak R. [Comparison of irrational beliefs, social support and social anxiety of girl students with employed mothers and unemployed among high school in Azhvaz (Persian)] [MSc. Dissertation]. Ahvaz: Islamic Azad University of Ahvaz; 2006.

[26] Woody D, Woody DJ. The significance of social support on parenting among a group of single, low-income, African American mothers. Journal of Human Behavior in the Social Environment 2007; 15(2-3):183-98. [DOI:10.1300/J137v15n02_11]

[27] Asgari P, Sharafoddin H. [Relationship of social anxiety, hope and social support with subjective well-being in complementary education students of Khoozestan Research's and Science Center (Persian)]. Journal of Social Psychology (New Findings in Psychology). 2009; 3(9):25-36.

[28] Tuckman BW. The development and concurrent validity of the procrastination scale. Educational and Psychological Measurement. 1991; 51(2):473-80. [DOI:10.1177/0013164491512022]

[29] Akinsola MK, Tella A, Tella A. Correlates of academic procrastination and mathematics achievement of university undergradu- 
ate students. Eurasia Journal of Mathematics, Science \& Technology Education. 2007; 3(4):363-70. [DOI:10.12973/ejmste/75415]

[30] Lee E. The relationship of motivation and flow experience to academic procrastination in university students. The Journal of Genetic Psychology. 2005; 166(1):5-15. [DOI:10.3200/GNTP.166.1.515] [PMID]

[31] Bayat M. [Standardized for Takman procrastination scale of students (Persian)] [MSc. Dissertation]. Roodehen: Roodehen University of Medical Sciences; 2002

[32] Broeck A, Vansteenkiste M, Witte H, Soenens B, Lens W. Capturing autonomy, competence, and relatedness at work: Construction and initial validation of the Work-related Basic Need Satisfaction scale. Journal of Occupational and Organizational Psychology. 2010; 83(4):981-1002. [DOI:10.1348/096317909x481382]

[33] Ghorbani N, Watson PJ. [Self-knowledge processes and rational and experiential information processing systems in Iran and the United States (Persian)]. Developmental Psychology. 2005; 5:3-14

[34] Marnat GG. Handbook of psychological assessment for clinical psychologists, Counselors and psychotherapists [Pashasharifi $\mathrm{H}_{\text {, }}$ Nikkhoo MR, Persian trans.]. Tehran: Sokhan; 2014. [PMID]

[35] Faramarzi f. [The effectiveness of life skills training on anxiety reduction and increase accountability on addicted to represent the well-being Soleiman Masjed (Persian)] [MSc. Dissertation]. Ahvaz: Islamic Azad University of Ahvaz; 2005.

[36] Megargee E.I. The California Psychological Inventory Handbook. San Francisco: Jossey-Bass Inc Pub, 1972.

[37] Pasha GhR, Goodarzian M. [The relationship between identity and moral growth with responsibility of students (Persian)]. Journal of Social Psychology. 2008; 2(8): 87-99.

[38] Atef Vahid MK, Nasr Esfahani M, Fattolahi P, Shojaie MR [Standardization of the Persian Version of the California Psychological Inventory (PCI) (Persian)]. Iranian Journal of Psychiatry and Clinical Psychology. 2006; 11(4):371-8.

[39] Tabachnick BG, Fidell LS. Using Multivariate statistics. Booston: Pearson Education; 2007. [PMCID]

[40] Hsu S, Chung Huang K, Sue Huang K. The relative study of social support, job satisfaction and wellbeing of military instructors: The case of high schools' military instructors in Yunlin, Chiayi and Tainan Districts of Taiwan. The Journal of International Management Studies. 2015; 10(1): 41-9.

[41] Asgari P, Naderi F, Hikel K. [The relationship between personality characteristics and social support and job satisfaction among female teachers City Ahvaz (Persian)]. Women and Culture. 2012; 1(1):69-80.

[42] Riggle RJ, Edmondson DR, Hansen JD. A meta-analysis of the relationship between perceived organizational support and job outcomes: 20 years of research. Journal of Business Research. 2009; 62(10):1027-30. [DOI:10.1016/j.jbusres.2008.05.003]

[43] Erdogan B, Enders J. Support from the top: Supervisors' perceived organizational support as a moderator of leader-member exchange to satisfaction and performance relationships. Journal of Applied Psychology. 2007; 92(2):321-30 [DOI:10.1037/00219010.92.2.321] [PMID]

[44] Hosseinzadeh AH, Bahrami Nezad Z, Frootan Kia Sh. [The effect of social support, emotional and strong links to public health
(The case of high school students in the boarding area Sardasht Dezful) (Persian)]. Journal of Social Sciences. 2012; 17(6): 146-64.

[45] Mazlo A. Motivation and personality [Rezvani A, Persian trans]. Mashhad: Astan-e Qods-e Razavi Pub; 2011.

[46] Kwong EW, Kwan AY. Stress-management methods of the community-dwelling elderly in Hong Kong: Implications for tailoring a stress-reduction program. Geriatric Nursing. 2004; 25(2):102-6. [DOI:10.1016/j.gerinurse.2004.02.007] [PMID]

[47] Karimi E, Mousavi M, Aiieni H. [The relationship between accountability and social anomie (negligence) among Employees of Rural Cooperative in Kurdistan (Persian). Paper presented at the $3^{\text {rd }}$ International Conference on Management, Accounting and Knowledge-Based Economy. 14 September 2017; Trhran, Iran.

[48] Kim KR, Seo EH. The relationship between procrastination and academic performance: A meta-analysis. Personality and Individual Differences. 2015; 82:26-33. [DOI:10.1016/j.paid.2015.02.038]

[49] Nasiri F. [The relationship between perceived organizational support urban management with social responsibility and job performance among Municipal employees (Persian)]. Journal of Urban Economics and Management. 2014; 2(8):97-110.

[50] Lonergan J. Locus of control as moderator of the relationship between job characteristics and procrastination at work $[\mathrm{PhD}$ dissertation]. California: California State University, Long Beach; 1998

[51] Vallerand RJ, Pelletier LG, Blais MR, Briere NM, Senecal C, Vallieres EF. The academic motivation scale: A measure of intrinsic, extrinsic, and amotivation in education. Educational and Psychological Measurement. 1992; 52(4):1003-17. [DOI:10.1177/0013164 492052004025]

[52] Rio JM. Motivational and emotional [Seid Mohammadi Y, Persian trans.]. Tehran: Virayesh; 2015. 